\title{
New results on precautionary saving and nonlinear risks
}

\author{
Claudio Bonilla ${ }^{1}$ (D) Marcos Vergara $^{2}$
}

Received: 30 June 2021 / Accepted: 16 November 2021 / Published online: 3 January 2022

(C) The Author(s), under exclusive licence to Springer-Verlag GmbH Austria, part of Springer Nature 2021

\begin{abstract}
We study precautionary saving in a two-period model that allows for nonlinear risks and nonseparable preferences. Permitting nonlinear risk effects is important because they are common in the developing world or when worldwide shocks hit economies, like the COVID-19 pandemic. Allowing nonseparable preferences is also important because they admit the incorporation of intergenerational transfer, habit persistence and other specific features of intertemporal decision making. We decompose the risk shock using Davis's (Int Econ Rev 30(1):131-136, 1989) compensation method and analyze the income and substitution effect of an increase in risk. We prove that the substitution effect is always negative and, therefore, the income effect must be positive and larger in size to have a precautionary net effect. We then apply the method to various sources of risk, such as income, interest rate and wealth risk. We analyze the magnitude of each effect and find the conditions required to guarantee precautionary saving in each case. Our results are presented as signs of covariances, which provides a new perspective on precautionary saving.
\end{abstract}

Keywords Precautionary saving - Nonlinear risk · Nonseparable preferences · Increases in risk $\cdot$ Mean-preserving spreads

JEL Classification E21 · D81 · D11

Claudio Bonilla

cbonilla@fen.uchile.cl

1 School of Economics and Business, University of Chile, Av. Diagonal Paraguay 257,

Suite 1906, Santiago de Chile, Chile

2 School of Economics and Business, Universidad del Desarrollo, Santiago de Chile, Chile 


\section{Introduction}

When an expected utility decision-maker faces an increase in future risk, he changes his optimal saving decision in order to better accommodate his intertemporal consumption and risk exposure. The usual concept that arises in this type of analysis is the precautionary saving effect, which is the extra saving produced in response to the increase in future risk. Under additive risks, this effect is guaranteed as long as the third derivative of the utility function is positive (Leland 1968; Sandmo 1970; Kimball 1990).

Most of the literature on precautionary saving assumes additively separable intertemporal utility functions and linear risks that are most frequently associated with a risk shock to future wages or to the interest rate (Menegatti 2007; Baiardi et al. 2014). In particular for interest rates, Eeckhoudt and Schlesinger (2008) show that, when there is an increase in the Nth-degree risk in the return on saving, we observe a precautionary effect as long as the measure of relative $(N+1)$ th-degree risk aversion exceeds $N$. This result is a generalization of the idea that, for secondorder risk increases, the condition that ensures precautionary saving is that the partial prudence index is greater than 2 (Rothschild and Stiglitz 1971). Recently, Magnani (2017) and Wong (2019) provided a new interpretation of these "threshold conditions" for the cases of second- and higher- order risk increases that enrich the intuition. However, all these previous results are based on separable preferences and linear risk structures. In this paper, we advance the literature by relaxing both of these assumptions. This allows us to obtain new conditions, which help explain empirical findings that seem to be at odds with precautionary saving (Dercon 2005).

It is easy to find contexts where preferences are not intertemporally separable and risk structures are not linear. For example, heterogeneous agents with intergenerational transfer (which is a common feature of wealthy households) imply nonseparable intertemporal preferences (Ponce and Yuki 2006). We also have the case of ambiguous environments in dynamic settings (Gomes 2014; Peter 2019), or of habit-persistent models where past consumption has a direct effect on present utility (Díaz et al. 2003). Nonlinear risk effects are also not difficult to find in real world examples. For instance, Gunning (2010) describes developing economies that present nonlinear risk shocks due to credit market constraints and unsolved asymmetric information problems. In the insurance literature, we also find nonlinear effects in health care applications (Echazu and Nocetti 2013), where the health production function incorporates nonlinear cross effects between the marginal productivity of investment in health and the random variable that captures the risk effects. We also find nonlinear effects in self-insurance with technology risk (Li and Peter 2021) when decision-makers lack precise information about the benefits of risk mitigation. For these cases and others, a positive third derivative of the utility function (known as the prudence property) may not be enough to secure the precautionary effect (Eeckhoudt and Schlesinger 2008; Vergara 2017; Baiardi et al. 2020; Vergara and Bonilla 2021). Therefore, modeling nonseparable preferences and nonlinear risks are the key to understand decision-making in those contexts. 
To study the effect of risk on saving, we decompose the risk shock using Davis's (1989) compensation method to distinguish between income and substitution effects of a risk increase. In our context, the income effect induces the prudent agent to save more in order to increase his expected income in the second period, when the uncertainty is present, whereas the substitution effect induces the risk-averse agent to save less in order to avoid the effect of an increase in risk. We show that a nonlinear risk shock amplifies both effects, and it also generates new effects that make it more difficult to sign the net effect on saving. We also show that, for the linear multiplicative risk case (interest rate and wealth risk), the income and substitution effects are slightly different from those found in Dardanoni (1988) for a utility function with two arguments and in Baiardi et al. (2020) for intertemporally separable preferences.

We share the perspective of Gunning (2010), who analyzed various sources of risk and studied labor, interest rate and wealth risk, and suggested that individuals in developing countries are exposed to great uninsured risks that are mostly nonlinear and have different effects on optimal decisions. Our paper is also related to Snow (2003) and Machina and Pratt (1997). The former decomposes a mean-preserving spread into a substitution and income effect but only for the case of an additively separable utility function with no specific applications whatsoever, and the latter extends the Rothschild and Stiglitz (1970, 1971) characterization of a meanpreserving spread to a more general definition with an extension to second-order stochastic dominance. In this paper we develop specific applications and connect our results with recent literature on decision-making under risk through a perspective of using the signs of covariances that appear in optimal saving problems. This covariance representation has the advantage of incorporating not only the sign of the effects but also the magnitude of the slope of change. It is, therefore, potentially more informative than the usual representation of the typical comparative static analysis.

It is important to mention that we provide a theoretical grounding for the case in which increases in risk induce reductions in saving. This empirical regularity observed in the developing world (Dercon 2005 and Elbers et al. 2007) ${ }^{1}$, can now be explained under nonlinear shocks when comparing the effect of the income and substitution effect revealed using Davis (1989)'s method.

\section{The two-period model}

\subsection{Notation}

We consider a two-period consumption model with a deterministic endowment income $w_{1}$ in the first period but an uncertain total income in the second period. The consumer has a utility function $u\left(c_{1}, c_{2}\right)$, which is assumed to be continuous,

\footnotetext{
1 Elbers et al. (2007) looked at the situation in Zimbabwe, a low-income country with significant credit market constraints and an underdeveloped financial system, and discovered that increases in risk led to reductions in growth due to a drop in capital accumulation and saving. Risk increases did not induce a precautionary effect on saving and investment, highlighting the need to account for nonlinear effects.
} 
increasing and concave in each argument, and at least three times differentiable. That is, $u_{1}$ and $u_{2}$ are positive while $u_{11}$ and $u_{22}$ are negative, where the subscripts denote partial derivatives. We assume that $u_{12}=u_{21} \geq 0$, meaning that consumption levels in the first and second periods interact as complements. ${ }^{2}$ Also, $c_{1}=w_{1}-s$ and $c_{2}=g\left(\theta, w_{2}, s\right)$, where $\theta$ represents a stochastic shock that affects total income in the second period, $w_{2}$ is a non-stochastic exogenous variable (second-period deterministic endowment) and $s$ denotes saving. In order to avoid explosive behavior and to make sure that we have a concave problem with interior solution, we provide natural assumptions for the shape of function $g$. In particular, we assume that the function $g$ is increasing in $w_{2}$ and increasing and concave in $s$ ( $g_{s}>0$ and $g_{s s} \leq 0$ ), which implies that saving raises second-period consumption at a decreasing rate. The function $g$ is also increasing and concave in $\theta\left(g_{\theta}>0\right.$ and $\left.g_{\theta \theta} \leq 0\right)$, which means that a positive shock of the random variable is beneficial to the decisionmaker at a decreasing rate. Finally, $g_{s}$ is increasing and concave in $\theta$; that is, $g_{s} \geq 0$ and $g_{s \theta \theta} \leq 0$, which implies that the random variable raises the incentive to save at a decreasing rate. ${ }^{3}$ It is worth noting that all of these assumptions regarding the curvature of $g$ are intuitive in economic terms, and they also correctly represent a positive random shock with no explosive higher-order effects, thereby ensuring a concave and tractable program.

Let $F(\theta, \tau)$ be the cumulative distribution function of $\theta$ with support in the interval $[a, b]$, where $\tau$ is a parameter whose shift represents changes in risk, like in Diamond and Stiglitz (1974). The consumer seeks the optimal savings $s^{*}$ that maximize the intertemporal utility of consumption, which is achieved by choosing $s^{*}(\tau)$, such that

$$
s^{*}(\tau)=* \operatorname{Arg} \max _{s}\left\{E u\left(c_{1}, c_{2}\right)=\int_{a}^{b} u\left(w_{1}-s, g\left(\theta, w_{2}, s\right)\right) d F(\theta, \tau)\right\}
$$

The first- and second-order conditions of the consumer's problem are:

$$
\int_{a}^{b}\left[-u_{1}+u_{2} g_{s}\right] d F(\theta, \tau)=-E u_{1}+E u_{2} g_{s}=0
$$

\footnotetext{
2 The classical articles of Leland (1968), Sandmo (1970) and Dardanoni (1988) consider nonseparable utility functions in their analyses. However, it has been customary in intertemporal economics to assume time-separable utility functions because of their simplicity and tractability (Baiardi et al. 2020). The problem with this assumption is that it rules out phenomena like intergenerational transfers or habit persistence (Díaz et al. 2003) and the positive effects of government spending on private consumption (Bilbiie 2009), which implies a positive correlation between first and second-period consumption (Gollier 2001).

${ }^{3}$ For example, Gunning (2010) represents a capital income risk by making $c_{2}=g\left(\theta, w_{2}, s\right)=w_{2}+(1-\delta) s+\theta h(s)$, where $w_{2}$ is the expected second-period labor income, $(1-$ $\delta) s$ is the expected value of physical assets, $\delta \in[0,1]$ is the depreciation rate and $h(s)$ is the expected value of capital income. The function $h(s)$ is increasing and concave, with $h(0)=0$. If $\delta=1$ and $h(s)=r s$, second-period consumption is $c_{2}=g\left(\theta, w_{2}, s\right)=w_{2}+\theta r s$, which is the usual interest rate risk case analyzed in the literature (Baiardi et al. 2020). Note that, in the world of Gunning (2010), there is space for a nonlinear relationship between consumption and saving, while the shocks are linear.
} 


$$
\int_{a}^{b}\left[u_{11}-2 u_{12} g_{s}+u_{22} g_{s}^{2}+u_{2} g_{s s}\right] d F(\theta, \tau)<0
$$

For ease of exposition, we will use the following definitions in the rest of the paper:

$$
\begin{gathered}
U(s, \theta)=u\left(w_{1}-s, g\left(\theta, w_{2}, s\right)\right) \\
U_{s}(s, \theta)=-u_{1}+u_{2} g_{s} \\
U_{s \theta}(s, \theta)=-u_{12} g_{\theta}+u_{22} g_{\theta} g_{s}+u_{2} g_{s \theta} \\
U_{s \theta \theta}(s, \theta)=-u_{122} g_{\theta}^{2}-u_{12} g_{\theta \theta}+u_{222} g_{\theta}^{2} g_{s}+u_{22} g_{\theta \theta} g_{s}+u_{22} g_{\theta} g_{s \theta}+u_{22} g_{\theta} g_{s \theta}+u_{2} g_{s \theta \theta}
\end{gathered}
$$

Next, we will study how changes in risk affect saving under various sources of risk. We will start with the traditional definition of a risk increase from Diamond and Stiglitz (1974), and then we will apply the compensation method to better understand risk increases in our applied examples, which include special cases of nonlinear risk.

\subsection{Risk changes}

Following Diamond and Stiglitz (1974) we define an increase in $\tau$ as a first-order risk increase if

$$
F_{\tau}(\theta, \tau) \geq 0
$$

and $F_{\tau}(a, \tau)=F_{\tau}(b, \tau)=0$.

Alternatively, an increase in $\tau$ represents a second-order risk increase (meanpreserving spread) if

$$
\int_{a}^{b} F_{\tau}(\theta, \tau) d \theta=0
$$

and

$$
T(\theta, \tau)=\int_{a}^{\theta} F_{\tau}(z, \tau) d z \geq 0 \text { for all } a \leq \theta \leq b
$$

and also, $T(a, \tau)=T(b, \tau)=0$. Condition (9) indicates that both distributions have the same mean, and condition (10) is the single-crossing property of a mean-preserving spread.

Proposition 1 (Conditions for the Precautionary Effect) Let $s^{*}(\tau)$ be the optimal level of saving that maximizes (1). Then, we have the following two results:

(i) If a shift in $\tau$ represents a first-order risk increase, then $s^{*}$ increases if $U_{s \theta}<0$. 
(ii) If a shift in $\tau$ represents a mean-preserving spread, then $s^{*}$ increases if $U_{s \theta \theta}>0$.

Proof (i) Using the implicit function theorem in the first-order condition (2), we obtain:

$$
\frac{d s^{*}}{d \tau}=-\frac{\int_{a}^{b} U_{s} d F_{\tau}(\theta, \tau)}{S O C}
$$

where SOC stands for the second-order condition, which is negative, as observed in (3). Therefore, the sign of $\frac{d s^{*}}{d \tau}$ is the same as the sign of $\int_{a}^{b} U_{s} d F_{\tau}(\theta, \tau)$.

Now, integrating expression $\int_{a}^{b} U_{s} d F_{\tau}(\theta, \tau)$ by parts, we get $-\int_{a}^{b} U_{s \theta} F_{\tau} d \theta$, which is positive as long as $U_{s \theta}<0$, which guarantees the precautionary effect for a firstorder risk increase and completes part (i) of the proof.

(ii) Now integrating expression $-\int_{a}^{b} U_{s \theta} F_{\tau} d \theta$ by parts again, we get $\int_{a}^{b} U_{s \theta \theta} T(\theta, \tau) d \theta$, which is positive as long as $U_{s \theta \theta}>0$, which guarantees the precautionary effect for the mean-preserving spread and completes part ii) of the proof.

\section{A specific definition of a mean-preserving spread}

Let us write the random variable $\theta$ as

$$
\theta=\mu_{\theta}+\gamma \epsilon
$$

where $\mu_{\theta}$ is the mean of $\theta, \gamma$ is a positive scalar that represents a spread parameter and $\epsilon$ is a zero-mean random variable. Equation (12) represents a parametric class of mean-preserving spreads. This class was introduced by Sandmo (1970) in his seminal contribution of saving under uncertainty. Later, Sinn (1983) and Meyer (1987) use it to define a location-scale family of distribution that allows mapping from the expected utility into the mean-variance space. Equation (12) is also related to the notion of "strong increases in risk" by Meyer and Ormiston (1985) as an increase in $\gamma$ extends the support of $\theta$ and allows us to go beyond the usual comparative statics of risk introduction to a comparative static considering increases in risk for already risk-averse agents. Note that an increase in $\gamma$ generates a meanpreserving spread in $\theta$. In this case, the consumer's intertemporal saving problem becomes

$$
s^{*}(\gamma)=* A \operatorname{Arg} \max _{s}\left\{E u\left(w_{1}-s, g\left(\mu_{\theta}+\gamma \epsilon, w_{2}, s\right)\right\}\right.
$$

Proposition 2 Let $s^{*}(\gamma)$ be the optimal level of saving that maximizes (13). A meanpreserving spread (increase in $\gamma$ ) induces an increase (decrease) in $s^{*}$ as long as $\operatorname{cov}\left(U_{\theta s}, \epsilon\right)>(<) 0$.

Proof By implicitly differentiating the first order condition derived from problem (13) we obtain: 


$$
\frac{d s^{*}}{d \gamma}=-\frac{-E u_{12} g_{\theta} \epsilon+E u_{22} g_{s} g_{\theta} \epsilon+E u_{2} g_{s \theta} \epsilon}{S O C}=-\frac{\operatorname{cov}\left(U_{\theta s}, \epsilon\right)}{S O C}
$$

which proves that, as long as $\operatorname{cov}\left(U_{\theta s}, \epsilon\right)>0$, we have a precautionary effect.

Note that $\operatorname{cov}\left(U_{\theta s}, \epsilon\right)=\operatorname{cov}\left(\left(-u_{12}+u_{22} g_{s}\right) g_{\theta}, \epsilon\right)+\operatorname{cov}\left(u_{2} g_{\theta s}, \epsilon\right)$. Below, we will show that the second covariance can be linked to the substitution effects by applying Davis (1989)'s compensation method, and following the terminology of the certainty case, we may call the first covariance "the income effect".

\section{The compensation method}

In many economic models, the results of comparative static exercises are defined by income and substitution effects, and most of the time they have opposing effects, resulting in possible indeterminacies.

The compensation method we use in this article is based on Davis (1989)'s paper, which in turn, is based on Sandmo's intuitive idea that a mean-preserving spread can be decomposed into income and substitution effect. Therefore, the compensation method (in a risky context) pursues the amount (given to or taken from $w_{2}$ ) sufficing to obtain the same amount of $c_{2}^{*}$ if the choice of saving remains $s^{*}$.

When the consumer experiences a mean-preserving spread, precautionary saving will induce an increase in saving. This increase in saving will, in turn, induce an increase in expected $c_{2}^{*}$. Therefore, the compensation method will provide a negative substitution effect (to go back to initial $c_{2}^{*}$ ). Consequently, the income effect must be positive and larger in size than the substitution effect to result in precautionary saving.

Let us assume that $w_{2}=\varphi(\gamma)$. The consumption in the second period is represented by

$$
c_{2}=g\left(\mu_{\theta}+\gamma \epsilon, \varphi(\gamma), s^{*}\right)
$$

The compensation method implies the following compensation condition or random rule:

$$
\frac{\partial c_{2}}{\partial \gamma}=g_{\theta} \epsilon+g_{w_{2}} \varphi_{\gamma}=0
$$

Solving for $\varphi_{\gamma}$ we get:

$$
\varphi_{\gamma}=-\frac{g_{\theta} \epsilon}{g_{w_{2}}}
$$

In order to obtain the substitution effect, we plug $\varphi(\gamma)$ into the first order condition

(2) and apply the implicit function theorem to get 


$$
\left.\frac{d s^{*}}{d \gamma}\right|_{c o m p}=-\frac{\left(-E u_{12}+E u_{22} g_{s}\right)\left(g_{\theta} \epsilon+g_{w_{2}} \varphi_{\gamma}\right)+E u_{2}\left(g_{s \theta} \epsilon+g_{s w_{2}} \varphi_{\gamma}\right)}{S O C}
$$

Considering the compensation rule (16) and following Davis's (1989) assumption that $g_{s w_{2}}=0$, Eq. (18) is reduced to

$$
\left.\frac{d s^{*}}{d \gamma}\right|_{\text {comp }}=-\frac{E u_{2} g_{s \theta} \epsilon}{S O C}=-\frac{\operatorname{cov}\left(u_{2} g_{s \theta}, \epsilon\right)}{S O C}
$$

and, consequently, the substitution effect is linked to the sign of $\operatorname{cov}\left(u_{2} g_{s \theta}, \epsilon\right)$.

Now recall that Eq. (14) provides the total effect on $s^{*}$ under a mean-preserving spread. In particular, a positive sign of $\operatorname{cov}\left(U_{\theta s}, \epsilon\right)=\operatorname{cov}\left(\left(-u_{12}+u_{22} g_{s}\right) g_{\theta}, \epsilon\right)+$ $\operatorname{cov}\left(u_{2} g_{\theta s}, \epsilon\right)$ guarantees precautionary savings. In addition, the compensation method provides the substitution effect by $\operatorname{cov}\left(u_{2} g_{\theta s}, \epsilon\right)$. Therefore, $\operatorname{cov}\left(\left(-u_{12}+\right.\right.$ $\left.\left.u_{22} g_{s}\right) g_{\theta}, \epsilon\right)$ is the income effect. The sign of each covariance component is obtained by differentiating the following expressions:

$$
\frac{\partial\left(-u_{12}+u_{22} g_{s}\right) g_{\theta}}{\partial \epsilon}=\left\{-u_{122} g_{\theta}^{2}-u_{12} g_{\theta \theta}+u_{222} g_{\theta}^{2} g_{s}+u_{22} g_{\theta \theta} g_{s}+u_{22} g_{\theta} g_{\theta s}\right\} \gamma
$$

$$
\frac{\partial u_{2} g_{s \theta}}{\partial \epsilon}=\left\{u_{22} g_{\theta} g_{s \theta}+u_{2} g_{s \theta \theta}\right\} \gamma
$$

Note that the substitution effect is actually negative, which is given by the sign of (21). Also, by adding (20) and (21) and dividing by $\gamma$, we get

$$
U_{s \theta \theta}=\underbrace{\left\{-u_{122} g_{\theta}^{2}-u_{12} g_{\theta \theta}+u_{222} g_{\theta}^{2} g_{s}+u_{22} g_{\theta \theta} g_{s}+u_{22} g_{\theta} g_{s \theta}\right.}_{\text {Income effect }}+\underbrace{u_{22} g_{\theta} g_{s \theta}+u_{2} g_{s \theta \theta}}_{\text {Substitution effect }}
$$

and we already know from previous propositions that, as long as $U_{s \theta \theta}>0$, precautionary saving is guaranteed under a mean-preserving spread.

We can see from expression (22) that risk aversion and prudence are insufficient to ensure the existence of precautionary saving. To better understand our findings, let us rewrite Eq. (14) by separating the income and substitution effects as follows:

$$
\frac{d s^{*}}{d \gamma}=-\frac{\operatorname{cov}\left(\left(-u_{12}+u_{22} g_{s}\right) g_{\theta}, \epsilon\right)}{S O C}+\frac{-\operatorname{cov}\left(u_{2} g_{\theta s}, \epsilon\right)}{S O C}
$$

It is worth noting that, according to (21), the substitution effect is always negative, whereas the income effects can be either negative or positive, given expression (20). As a result, our formulation implies that $\frac{d s^{*}}{d \gamma}$ can be positive with precautionary saving or negative, as suggested by Dercon (2005), in the case of developing countries.

In our model, an uncertainty shock is translated into a mean-preserving spread via $\mu_{\theta}+\gamma \epsilon$, resulting in an increase in the spread parameter $\gamma$. The substitution 
effect observed in (21) (which is derived from the compensation method) reflects the economic agent's desire to reduce risk exposure by decreasing saving in the face of an increase in risk. The substitution effect is always negative, given risk aversion $\left(u_{22}<0\right)$, and a plausible assumption on the curvature of the function $g\left(g_{s \theta \theta}<0\right.$, explained in the previous section), as shown in (21). The income effect, on the other hand, represents and increases the riskiness of $c_{2}^{*}$, which translates into a reduction in the certainty equivalent of $c_{2}^{*}$, which has both positive and negative effects on saving, as shown by (20). Part of the positive effect is due to prudence $\left(u_{222} g_{\theta}^{2} g_{s}\right)$ and part of the negative effect is due to the nonlinear effects derived by function $g$ (the component $u_{22} g_{\theta} g_{\theta s}$ or $-u_{12} g_{\theta \theta}$, for example). As a result, we cannot rule out any possible final effect on saving when we consider both the substitution and income effect in our nonseparable and nonlinear model. Under a positive shock of uncertainty, we may experience the traditional precautionary effect or, alternatively, we may also experience a decrease in saving, which is, as we explained earlier, an empirical fact found in some specific circumstances.

Table 1 summarizes the income and substitution effects for different types of utility functions and risks.

At this point, it is important to mention that $g_{\theta \theta}$ and $g_{s \theta \theta}$ allow having nonlinear risks in our analysis. As we mentioned before, nonlinearities are often present in developing economies where relevant risks are uninsured (Gunning 2010) and increases in risk have been proven to decrease saving instead of inducing a precautionary effect (Dercon 2005; Elbers et al. 2007). Note, then, that cases like the ones described above - previously considered anomalies, from the traditional perspective of precautionary saving- are now incorporated into our general formulation.

Nonlinear risk also allows the theorization of new risks that are not commonly treated in the economics literature, like the risk of COVID-19 and its devastating economic consequences worldwide. In the end, Eq. (22) is a general expression that captures not only the traditional risk analysis commonly observed in the literature, but also new risks, with more complex functional forms and cross-related effects on saving.

In what follows, we will apply our general formulation to various sources of risk and define conditions for precautionary saving in each case. We connect these applications with previous literature and show that this general formulation

Table 1 Precautionary effect

\begin{tabular}{lll}
\hline Cases & Income effect & Substitution effect \\
\hline Additively separable utility & $u_{222} g_{\theta}^{2} g_{s}+u_{22} g_{\theta \theta} g_{s}+u_{22} g_{\theta} g_{\theta s}$ & $u_{22} g_{\theta} g_{\theta s}+u_{2} g_{s \theta \theta}$ \\
Linear risk & $-u_{122} g_{\theta}^{2}+u_{222} g_{\theta}^{2} g_{s}+u_{22} g_{\theta} g_{\theta s}$ & $u_{22} g_{\theta} g_{\theta s}$ \\
Additively separable utility and linear risk & $u_{222} g_{\theta}^{2} g_{s}+u_{22} g_{\theta} g_{\theta s}$ & $u_{22} g_{\theta} g_{\theta s}$ \\
Additive risk & $-u_{122} g_{\theta}^{2}+u_{222} g_{\theta}^{2} g_{s}$ & 0 \\
Additively separable utility and additive risk & $u_{222} g_{\theta}^{2} g_{s}$ & 0 \\
\hline
\end{tabular}


incorporates the known results from precautionary saving but also provides space for nonlinear shocks and specific non-conventional types of risk.

\section{Applications}

In this section we will study three sources of risk: classic labor income risk with a linear and additive shock; the case of interest-rate risk with a multiplicative and linear shock, like in Eeckhoudt and Schlesinger (2008); and finally, a wealth risk shock as in Gunning (2010). ${ }^{4}$

\subsection{Labor income risk}

Let us assume that second-period consumption is represented by

$$
c_{2}=g\left(\theta, w_{2}, s\right)=w_{2} \theta+s R
$$

where $\theta=\mu_{\theta}+\gamma \epsilon$ and $R$ is the non-random gross interest rate. In this case, $U(s, \theta)=u\left(w_{1}-s, w_{2} \theta+s R\right)$, and since in this case $g_{\theta}=w_{2}, g_{s}=R$ and $g_{\theta s}=g_{\theta \theta s}=g_{\theta \theta}=0$, then $\operatorname{cov}\left(U_{s \theta}, \epsilon\right)=\operatorname{cov}\left(\left(-u_{12}+u_{22} R\right) w_{2}, \epsilon\right)$. Therefore, the precautionary effect is, in this instance, determined only by the income effect. Note that $\operatorname{cov}\left(\left(-u_{12}+u_{22} R\right) w_{2}, \epsilon\right)>0$ if and only if $\frac{\partial U_{s \theta}}{\partial \epsilon}=\left(-u_{122}+u_{222} R\right) w_{2}^{2} \gamma>0$. If $u\left(c_{1}, c_{2}\right)$ is additively separable $\left(u_{122}=0\right)$, then precautionary saving takes place whenever the decision-maker is prudent, i.e. $u_{222}>0$.

\subsection{Interest-rate risk}

Let us assume that consumption in the second period is represented by the following equation:

$$
c_{2}=g\left(\theta, w_{2}, s\right)=w_{2}+s \theta R
$$

where $g_{s}=\theta R, g_{\theta}=s R, g_{\theta s}=R$ and $g_{\theta \theta}=g_{\theta \theta s}=0$. In this case $U(\theta, s)=$ $u\left(w_{1}-s, w_{2}+s \theta R\right)$ and $\operatorname{cov}\left(U_{\theta s}, \epsilon\right)=\operatorname{cov}\left(\left(-u_{12}+u_{22} \theta R\right) s R, \epsilon\right)+\operatorname{cov}\left(u_{2} R, \epsilon\right)$. The precautionary effect is determined by both the income effect and the substitution effect. The substitution effect is negative because the sign of $\operatorname{cov}\left(u_{2} R, \epsilon\right)$ can be obtained from the sign of

$$
\frac{\partial u_{2} R}{\partial \epsilon}=u_{22} s R^{2} \gamma
$$

Therefore, to have a precautionary effect, the income effect must be positive and greater in magnitude than the substitution effect. This means that

\footnotetext{
$\overline{4}$ Following Gunning (2010), all the examples are cases of linear risk.
} 


$$
-u_{122}(s R)^{2} \gamma+u_{222}(s R)^{2} \gamma \theta R+u_{22} s R^{2} \gamma>-u_{22} s R^{2} \gamma
$$

By working on inequality (26) and assuming a separable utility function, we obtain the following condition to guarantee the precautionary effect

$$
\frac{-u_{222}}{u_{22}} s \theta R>2
$$

where $\frac{-u_{222}}{u_{22}} s \theta R$ is the partial prudence coefficient. ${ }^{5}$

\subsection{Wealth risk}

Following Gunning (2010) we define second-period consumption by

$$
c_{2}=g\left(\theta, w_{2}, s\right)=\theta\left(w_{2}+(1-\delta) s+h(s)\right)=\theta W
$$

where $W$ stands for total wealth, $(1-\delta) s$ the expected value of assets, and $h(s)$ is the expected value of capital income. The function $h(s)$ is increasing $\left(h^{\prime}>0\right)$ and concave $\left(h^{\prime \prime}<0\right)$, with $h(0)=0$. Also, $g_{s}=\theta W_{s}, g_{\theta}=W, g_{s \theta}=W_{s}$, where $W_{s}=$ $(1-\delta)+h^{\prime}$ and $g_{\theta \theta}=g_{\theta \theta s}=0$.

In this case, $\quad U(\theta, s)=u(w-s, \theta W)) \quad$ and $\operatorname{cov}\left(U_{s \theta}, \epsilon\right)=\operatorname{cov}\left(\left(-u_{12}+u_{22} \theta W_{s}\right) W, \epsilon\right)+\operatorname{cov}\left(u_{2} W_{s}, \epsilon\right)$. We know that we have a precautionary effect as long as $\operatorname{cov}\left(U_{s \theta}, \epsilon\right)>0$. In this case the substitution effect is negative because

$$
\frac{\partial u_{2} W_{s}}{\partial \epsilon}=u_{22} W_{s} \gamma W<0
$$

and just like in the previous case, to have a precautionary effect, the income effect must be positive and greater in magnitude than the substitution effect. This means that in this case we need

$$
\left[-u_{122} W \gamma+u_{222} \theta W_{s} W \gamma+u_{22} W_{s} \gamma\right] W>-u_{22} W_{s} \gamma W
$$

By working on inequality (30) and assuming separable utility function, we obtain the following condition to guarantee the precautionary effect

$$
\frac{-u_{222}}{u_{22}} \theta W=\frac{-u_{222}}{u_{22}} c_{2}>2
$$

where $\frac{-u_{222}}{u_{22}} c_{2}$ is the relative prudence coefficient.

Table 2 summarizes the income effect and the substitution effect for the three types of risk described above.

\footnotetext{
${ }^{5}$ Note that Dardanoni (1988) postulates that the income effect is $s^{2}\left(-u_{122}+u_{222} \theta R\right)$, while the substitution effect is $2 u_{22} s$. However, applying Davis' compensation method (1989), the income effect is $s^{2}\left(-u_{122}+u_{222} \theta R\right)+u_{22} s$ and the substitution effect is $u_{22} s$. When the function is additively separable, the literature states that the income effect is $u_{222} s \theta R$, while the substitution effect is $2 u_{22}$ (Baiardi et al. 2020). In our case, the income effect is $u_{222} s \theta R+u_{22}$ and the substitution effect is $u_{22}$. Therefore, previous literature on precautionary saving is biased in favor of the substitution effect.
} 
Table 2 Income and substitution effect for various sources of risk

\begin{tabular}{llll}
\hline Sources & $g\left(\theta, w_{2}, s\right)$ & Income effect & $\begin{array}{l}\text { Substitution } \\
\text { effect }\end{array}$ \\
\hline $\begin{array}{l}\text { Labor income } \\
\text { risk }\end{array}$ & $w_{2} \theta+s R$ & $\left(-u_{122}+u_{222} R\right) w_{2}^{2} \gamma$ & 0 \\
$\begin{array}{l}\text { Interest-rate risk } \\
\text { Wealth risk }\end{array}$ & $w_{2}+s \theta R$ & $\left(s^{2}\left(-u_{122}+u_{222} \theta R\right)+u_{22} s\right) R^{2} \gamma$ & $u_{22} s R^{2} \gamma$ \\
\hline
\end{tabular}

\section{Conclusion}

In this paper we developed a general formulation for the two-period model of saving and consumption under uncertainty, allowing for more general risk structures in the second-period uncertainty shock. We argue that nonlinear risk structures are a common feature in the developing world, as previous empirical literature suggests, and that they also appear when unexpected large-scale risks hit and affect the world economy as a whole. The most contemporaneous example of this is the COVID-19 pandemic and its resulting economic downturn where still do not even know the shape its recovery will take.

To dive into the effects of increases in risk on saving, we use Davis (1989)'s compensation method and observe that, when the consumer faces a mean-preserving spread, precautionary saving can be divided into an income and a substitution effect, and these effects can be represented by covariances. The substitution effect is negative, so the income effect must then be positive and larger in size than the substitution effect to induce increases in savings and the expected precautionary effect.

Three applications are developed in the paper. In particular, we study the effects of various sources of risk such as income, interest rate and wealth risk. We then define the conditions for each case to exhibit the precautionary effect.

Finally, when we go back to the classic set-up of the model and assuming additively separable utility functions, the conditions for precautionary saving relate to the concepts of prudence and relative prudence, which is consistent with current literature on saving under risk. Nevertheless, we discover that adding nonlinear effects into our generalized model allows for a more integral analysis of economic cases previously excluded under the traditional approach.

Acknowledgements We would like to thank the useful comments of the Editor and two anonymous referees. Any errors that remain are the sole responsibility of the authors. Marcos Vergara thanks the support of ANID, FONDECYT Iniciación Project Number 11170052. 


\section{References}

Baiardi D, Magnani M, Menegatti M (2020) The theory of precautionary saving: an overview of recent developments. Rev Econ Househ 18:513-542

Baiardi D, Magnani M, Menegatti M (2014) Precautionary saving under many risks. J Econ 113(3):211-228

Bilbiie F (2009) Nonseparable preferences, fiscal policy puzzles and inferior goods. J Money Credit Bank 41:443-450

Davis G (1989) Income and substitution effects for mean-preserving spreads. Int Econ Rev 30(1):131-136

Díaz A, Pijoan-Mas J, Rios-Rull J (2003) Precautionary savings and wealth distribution under habit formation. J Monet Econ 50:1257-1291

Dardanoni V (1988) Optimal choices under uncertainty: the case of two-argument utility functions. Econ J 98(391):429-450

Dercon S (2005) Risk, insurance and poverty: a review. In: Dercon S (ed) Insurance against poverty. Oxford University Press, Oxford, pp 9-37

Diamond PA, Stiglitz JE (1974) Increases in risk and in risk aversion. J Econ Theory 8(3):337-360

Echazu L, Nocetti D (2013) Priority setting in health care: disentangling risk aversion from inequality aversion. Health Econ 22:730-740

Eeckhoudt L, Schlesinger H (2008) Changes in risk and the demand for saving. J Monet Econ 55(7):1329-1336

Elbers C, Willen J, Kinsey B (2007) Growth and risk: methodology and micro-evidence. World Bank Econ Rev 21(1):1-20

Gunning J (2010) Risk and savings: a taxonomy. Econ Lett 107(1):39-41

Gollier C (2001) The economics of risk and time. The MIT Press

Gomes F (2014) A note on uncertainty in saving decisions: Can a naive strategy be optimal? Macroecon Dyn 18(6):1428-1435

Kimball MS (1990) Precautionary saving in the small and in the large. Econometrica 58(1):53-73

Leland HE (1968) Saving and uncertainty: the precautionary demand for saving. Q J Econ 82(3):465-473

Li L, Peter R (2021) Should we do more when we know less? The effect of technology risk on optimal effort. J Risk Insurance forthcoming

Machina M, Pratt J (1997) Increasing risk: some direct constructions. J Risk Uncertain 14:103-127

Magnani M (2017) A new interpretation for precautionary saving in the presence of an interest-rate risk. J Econ 120:79-87

Menegatti M (2007) A new interpretation for the precautionary saving motive: a note. J Econ 92(3):275-280

Meyer J, Ormiston M (1985) Strong increases in risk and their comparative statics. Int Econ Rev 26(2):425-437

Meyer J (1987) Two-moment decision models and expected utility maximization. Am Econ Rev 77:421-430

Peter R (2019) Revisiting precautionary saving under ambiguity. Econ Lett 174:123-127

Ponce I, Yuki K (2006) Savings, intergenerational transfers, and the distribution of wealth. Macroecon Dyn 10(3):371-414

Rothschild M, Stiglitz JE (1970) Increasing risk: I. A definition. J Econ Theory 2:225-243

Rothschild M, Stiglitz JE (1971) Increasing risk: II. It economics consequences. J Econ Theory 3:66-84

Sandmo A (1970) The effect of uncertainty on saving decisions. Rev Econ Stud 37(3):353-360

Sinn HW (1983) Economic decisions under uncertainty. North Holland, Amsterdam

Snow A (2003) Substitution and income effects for increases in risk. Econo Lett 79:313-317

Vergara M (2017) Precautionary saving: a taxonomy of prudence. Econ Lett 150:18-20

Vergara M, Bonilla C (2021) Precautionary saving in mean-variance models and different sources of risk. Econ Model 98:280-289

Wong KP (2019) An interpretation of the conditions for precautionary saving: the case of greater higherorder interest rate risk. J Econ 126:275-286

Publisher's Note Springer Nature remains neutral with regard to jurisdictional claims in published maps and institutional affiliations. 